\title{
Changing digoxin potency and cardiac mortality in England and Wales 1968-76
}

\author{
HUGH TUNSTALL-PEDOE \\ From the Cardiovascular Epidemiology Unit, Ninewells Hospital and Medical School, Dundee
}

SUMmaRY Data on monthly totals of cardiac deaths in England and Wales were examined in different ways to see whether there were any unexplained fluctuations in rates both at the time of an unplanned increase in the bioavailability and therefore the potency of the Lanoxin brand of digoxin in May 1972 and also when there was a coordinated increase in the bioavailability of other brands in October 1975. Despite advice to prescribers, dosages were not proportionately reduced in the 600000 patients who were on treatment. Monthly totals of deaths from cardiac causes were high through the summer of 1972 but not at the end of 1975 and the excess in 1972 seems to be related to a cold summer. There was no evidence of a consistent or specific effect of changes in digoxin potency, either beneficial or harmful, on deaths from all cardiac causes or in specific subgroups in which digoxin treatment was likely to be most common. Although major changes in digoxin potency in England and Wales did not seem to produce a repeatable effect on death rates, data from other countries should also be examined for evidence of such an effect.

Digitalis is an old established drug widely used in the form of digoxin for the control of atrial fibrillation and the treatment of congestive cardiac failure. ${ }^{12}$ Its therapeutic index, however, is low, individual dosage is variable, particularly in the elderly in whom it is often prescribed, and toxic effects such as anorexia and rhythm disturbances may be confused with the conditions it is used to treat. Its value in improving symptoms is accepted but it is not known whether its mass usage in the population is beneficial or harmful since it has never been the subject of a large scale controlled trial such as is used for new drugs; however, some small scale trials have been reported. ${ }^{34}$ But digoxin has been the subject of two natural experiments in the United Kingdom. In 1969 the potency of the leading proprietary brand, Lanoxin, was reduced by about half as a result of a change in manufacturing procedures which affected its bioavailability. This was followed in the summer of 1972 by an unplanned doubling of potency at a time when new tests of bioavailability were being intro-

Requests for reprints to Professor Hugh Tunstall-Pedoe, Cardiovascular Epidemiology Unit, Ninewells Hospital and Medical School, Dundee DD1 9SY.

Accepted for publication 26 April 1985 duced. Consequently a new standard for bioavailablity was set, and there was a planned synchronised increase in the potency of all other brands in October 1975.

A previous study explored what happened to dosage levels and prescribing habits over this period. ${ }^{5}$ An estimated 600000 patients were found to be on long term treatment including $6 \%$ of the population over 65 . Although mean daily dosages showed a steady decline in the period 1967-76, there was no sudden change in dosage at the time of the changes in digoxin potency. In contrast to the small scale controlled trials, the natural experiment therefore involved a study population of 600000 who underwent a sudden increase in the effective dosage in 1972 and 1975.

Because digoxin is used exclusively for heart disease most of those who died from toxicity or whose lives might have been prolonged by the increased effective dosage would probably have had a diagnosis of heart disease shown on the death certificate. I therefore decided to examine the monthly trends in deaths for England and Wales attributed to heart disease over several years with an a priori requirement that if an association with changes of digoxin potency was to be established any increase or decrease in deaths seen in the summer of 1972 should also be seen in the autumn of 1975 . 


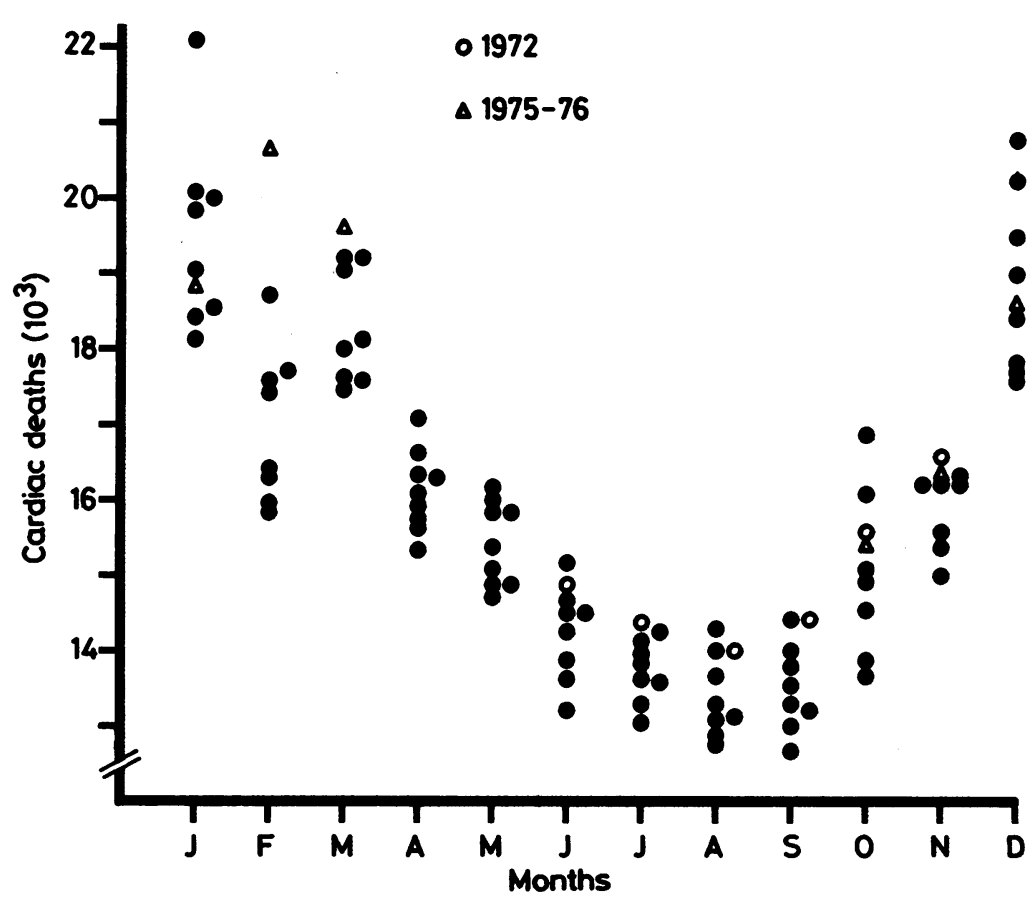

Fig. 1 Monthly totals of cardiac deaths 1968-76 showing how the six months from May 1972 and October 1975 compare with corresponding months in other years.

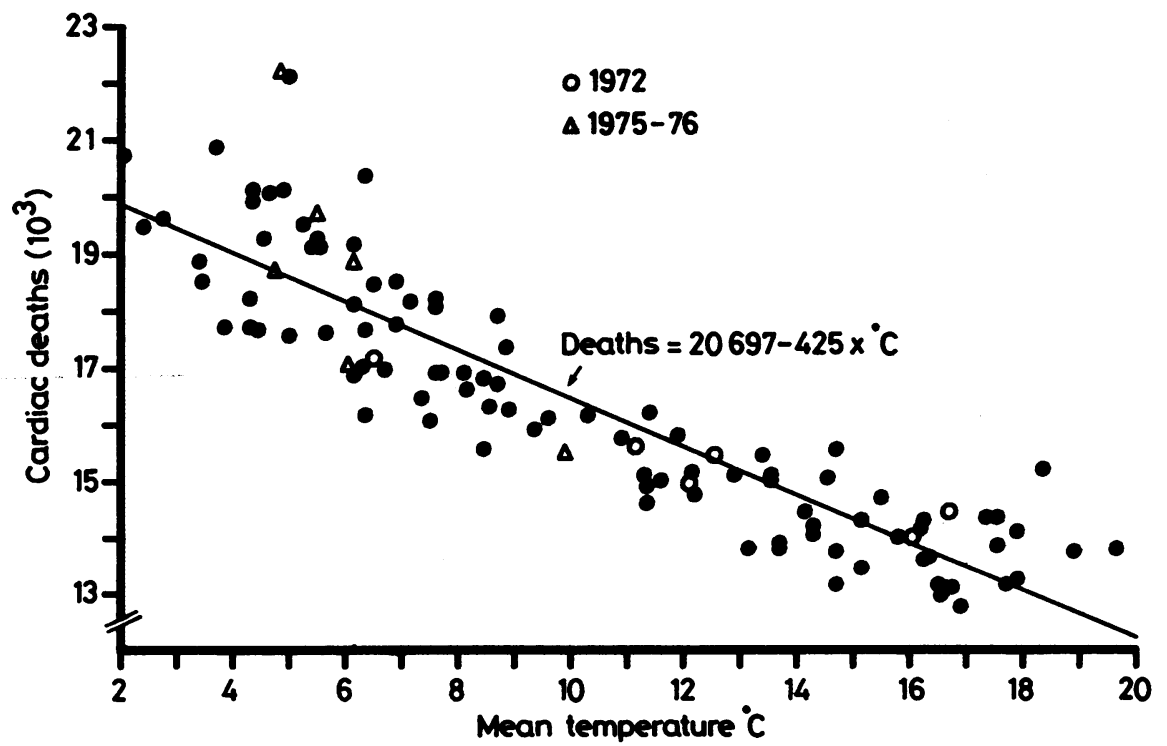

Fig. 2 Monthly totals of cardiac deaths 1968-76 corrected to 31 days and plotted against mean monthly temperature recorded at Kew. 


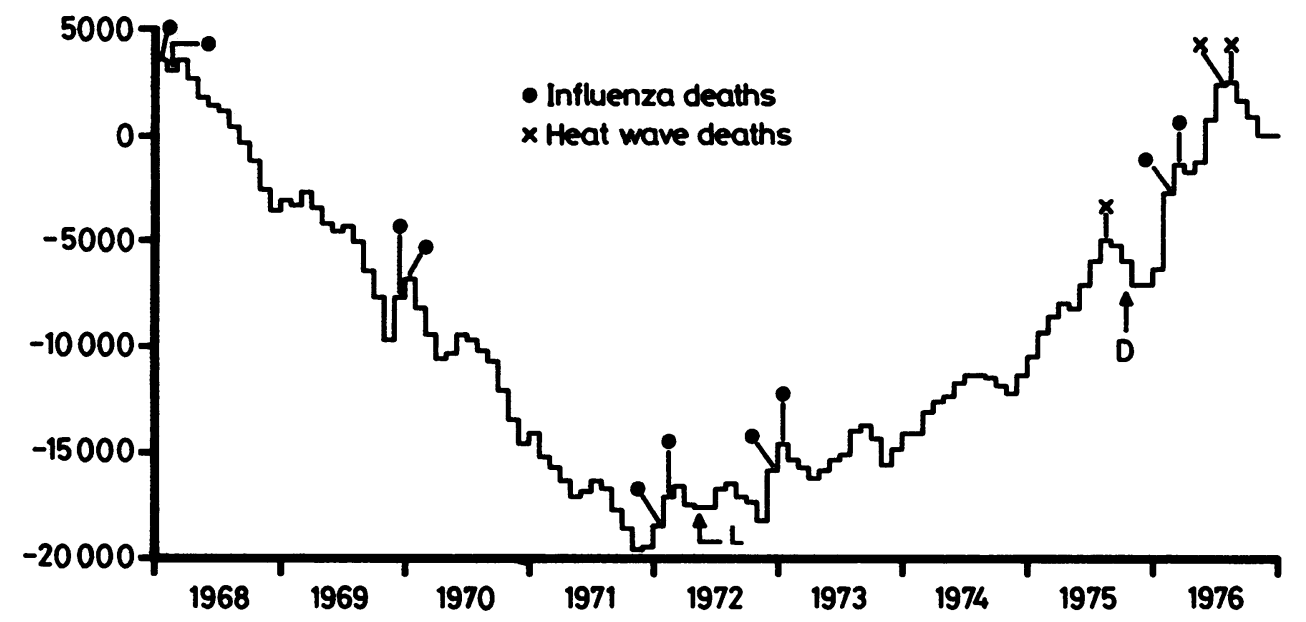

Fig. 3 Cumulative sum graph of observed minus expected cardiac deaths per month 1968-76 corrected for temperatures to show changes in trends. Months marked are those when influenza deaths exceeded 200 or when the mean temperature was above $18^{\circ} \mathrm{C}$. L, change in potency of Lanoxin brand of digoxin; $D$, general change in digoxin potency.

\section{Patients and methods}

Monthly totals of deaths in England and Wales were extracted from the Registrar General's publications from the years 1968 (when a new disease classification was introduced) to $1976 .{ }^{6}$ Different categories of deaths were looked at including deaths from all causes, respiratory deaths (International Classification of Disease rubrics 460-519), neoplasms (140-239), cardiovascular deaths (390-458), cardiac deaths (390-429), and different subgroups of the latter.

Cardiac deaths were subjected to special analyses. Because of the excess of deaths in the winter months, often accompanied by influenza epidemics and also related to the ambient temperature, the monthly totals for each year were displayed graphically, picking out the six months after the increase in Lanoxin potency in May 1972 and the increase in potency of other digoxin brands in October 1975 (Fig. 1). The number of deaths in each month was then adjusted upwards if necessary to correct for the shortfall from 31 days and they were plotted against the mean monthly temperature at Kew (Fig. 2).

A third technique was then used to explore these data in a different way. A regression equation was calculated to fit the data in Fig. 2 to relate numbers of cardiac deaths per month to the mean monthly temperature (corrected monthly death total $=20697-425 \times$ the mean monthly temperature in degrees $\mathrm{C}$ ). For each month from the beginning of 1968 the formula was used to calculate sequentially the expected number of deaths and any discrepancy (positive or negative) between that and the observed number was found. These were then put together by means of the cumulative sum (CUSUM) technique in which the discrepancies are added serially and plotted, so that a change in the gradient of the line shows that a new factor is operating (Fig. 3) ${ }^{7}$

Finally, I analysed the numbers of deaths per month in different cause groups. Periods of four months' duration after the two increases in potency were compared with the four month periods preceding the increase. Each monthly total in each category for February 1972 to May 1972 and for June 1975 to September 1975 was divided by the average number of deaths in that disease category in that calendar month during the years 1968 and 1976 to obtain a monthly score (this time uncorrected for temperature). The same calculation was done for the two four month periods June to September 1972 and October 1975 to January 1976. The sum of the scores for the eight post-potency increase months was then divided by the sum of the scores for the eight prepotency increase months to obtain a ratio (Table).

\section{Results}

A scatter diagram of number of cardiac deaths in each calendar month over the nine years is shown in Fig. 1. The results for June, July, August, and September 1972 are all rather high, there is no excess in October, but there is a rather high reading in November. The figures for October 1975 are average, 
Table Ratio of post-potency increase monthly scores to pre-potency increase monthly scores

\begin{tabular}{lcl}
\hline $\begin{array}{l}\text { Cause of death } \\
\text { (ICD) }\end{array}$ & $\begin{array}{l}\text { Number of deaths } \\
\text { in May 1972 }\end{array}$ & $\begin{array}{l}\text { Post-potency to } \\
\text { pre-potency ratio } \\
\text { of deaths }\end{array}$ \\
\hline All causes & 46834 & 0.97 \\
Neoplasms (140-239) & 10121 & 0.98 \\
Respiratory (460-519) & 5513 & 0.92 \\
Cardiovascular (390-458) & 24573 & 0.99 \\
Cardiac (390-429) & 15833 & 1.01 \\
Rheumatic heart (393-8) & 534 & 0.96 \\
Mitral valve (394) & 237 & 0.95 \\
Symptomatic heart (427) & 1076 & 0.98 \\
\hline
\end{tabular}

those for November are high, while those for December and January 1976 are below average; an influenza epidemic increased the numbers for the following February and March.

Figure 2, in which monthly rates are plotted against temperatures, shows the known association. Aberrant high peaks at the lower temperatures were related to influenza epidemics, and those at high temperatures to heat waves. The high monthly numbers in the summer of 1972 appear to be largely explained by the temperature. Nineteen seventy two had the coldest May, coldest June, an average July, the second coldest August, and the coldest September in the nine year period, based on the mean monthly figure recorded at Kew.

The cumulated sum graph (Fig. 3) which indicates trends after correction of data for temperature shows a tendency for deaths to decline in the late 1960 s, an accelerating decline at the end of 1970 through 1971 , and a change of trend at the beginning of 1972. Lanoxin potency was changed in May 1972 during a level phase and a positive gradient appears to start in late 1972. In contrast there was no obvious change in trends with the planned increase in potency of other digoxin brands in October 1975, which occurred during a period of five months when rates were lower than expected and was followed by an erratic year in 1976 when there was an influenza epidemic followed by summer heat waves. Both of these factors were associated with an excess of deaths in patients with heart disease.

The ratio of post-potency increase monthly scores to pre-increase scores (Table) shows no effect that seems to be specific to digoxin. Although the ratio for all cardiac deaths increased more than the ratios for deaths from all causes and for cardiovascular, respiratory, and neoplastic deaths, if this increase were the effect of a change in digoxin therapy there should have been an exacerbation in the categories of heart disease in which digoxin is most commonly used such as rheumatic heart disease, mitral heart disease, and the symptomatic heart disease category (which includes those patients in whom heart failure and arrhythmias were diagnosed without an underlying $\stackrel{c}{=}$ specific abnormality).

\section{Discussion}

Use of national mortality figures to determine the effects of medication is an unusual technique but has an important precedent. Asthma deaths were shown $\mathrm{s}$ to rise in young people at a time when new pressur- $\overrightarrow{0}$ ised aerosols that dramatically relieved symptoms $\overrightarrow{\vec{\omega}}$ were being widely introduced. ${ }^{8}$ Cardiac disease ${ }^{\omega}$ treated by digoxin is chronic and usually managed in the community and patients usually die while they of are on this medication. An increase (or decrease) in such deaths would not be detected by an individual i clinician or clinic unless the change was consid- $\omega$ erable. This is because a limited number of patients $ᄋ$ are treated in any one centre, sporadic fluctuations in deaths are normal, and there is not usually any for- $\infty$ mal surveillance of death rates. National mortality $\mathbb{D}$ figures cover the pooled experience of all the patients $\stackrel{\mathbb{D}}{3}$ and clinicians in the population with no loss to follow up. In this case 600000 patients were believed to be on treatment ${ }^{5}$ with a mean daily dose (all ages) of $\overrightarrow{0}$ about $380 \mu \mathrm{g}$ in 1972 and of $320 \mu \mathrm{g}$ in 1975 . If $1 \%$ of $\stackrel{0}{\circ}$ these patients had died or had had their death delayed as a result of the change in potency of the drug then the death rate would change by 6000 , but the chance of it coming to clinical attention in any single centre is very small.

There are several difficulties associated with the use of trends in cardiac deaths or indeed deaths from any cause. Over several years there are trends in numbers of deaths which are related to long term trends in disease rates (such as the decline in rheumatic heart disease) or to changes in the age structure of the population or to fashions in diagnosis. While such long term trends should not affect short term fluctuations, the latter are quite considerable. Rates are particularly likely to fluctuate in the winter when they are affected by the temperature, which can be allowed for (as demonstrated above), and by 욱 influenza outbreaks, which cannot since different $\rightarrow$ outbreaks have different virulence and may or may not differentially affect elderly patients with heart $N$ disease. It is difficult in retrospect to prove that any fluctuation is caused by medication because death 0 certificates do not record medication and there is no $\mathrm{\omega}$ routine surveillance of the mortality of all patients who are on established drugs. If there is a fluctuation in the death rate amounting to only a few per cent it $\Phi$ is not possible to identify which specific deaths were $\stackrel{+}{-}$ expected and which were exceptional without a very laborious and possibly inaccurate survey.

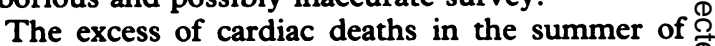
1972 was unusual in that it occurred at a time of the $\stackrel{D}{\varrho}$ 
year when death rates are usually stable, and a link with the change in Lanoxin potency must be suspected. There are several reasons, however, for thinking that this was unlikely. Firstly, the excess seems to be largely explained by the cold summer (Fig. 2) and, secondly, there was no corresponding increase in cardiac deaths after digoxin potency had increased in October 1975. In 1972 the manufacturer realised some time after stocks were first released to suppliers that potency had changed, but the new formulation would not have reached all patients at the same time, so that there would have been a delayed and progressive impact on patients as old stocks were used up and new ones were introduced. During this time, pharmacists were being encouraged to dispense other brands against general practitioner prescriptions for Lanoxin. Thus though we know what was being prescribed ${ }^{5}$ we do not know exactly what was being dispensed and when. The situation in October 1975 was much more straightforward. For the non-Lanoxin brands, the high potency variant was not released before October 1975 but was dispensed exclusively after that date so that the impact on the patient population would have been simultaneous. Unfortunately the proportion of patients on medication being supplied with nonLanoxin brands at this time is not known since many pharmacists dispensed Lanoxin against a generic prescription for digoxin and although it is claimed that Lanoxin had the bulk of the market in 1972, it was less popular afterwards and then began to recover its former position. The $a$ priori hypothesis of this investigation was that any effect of a change in digoxin potency would have been repetitive, but there is no evidence that the change of potency did have a consistent effect on cardiac deaths.

Two other features argue against the claim that digoxin was responsible for any of the excess of cardiac deaths in 1972. One is that while there was a peak in the broad category of cardiac deaths, this excess was less apparent in deaths attributed to rheumatic heart disease (and specifically mitral valve disease), for which a high proportion of patients would have been receiving large enough doses to control atrial fibrillation, and also in deaths from symptomatic heart disease which tend to occur in elderly patients being treated for congestive cardiac failure. Finally there was no clinical evidence of an epidemic of digitalis toxicity at this time. This contrasts with a report from Israel where a change in digoxin potency resulted in large numbers of patients being admitted to hospital with toxic effects. ${ }^{9}$ Although the major changes in Lanoxin potency in 1969 and 1972 were largely unremarked and led to little change in prescribing habits by clinicians, ${ }^{5}$ it would be surprising if an increase in deaths from digoxin toxicity in England and Wales could occur without prompting any comments from hospital doctors on the increase in morbidity, especially since such observations were being reported in other countries.

These observations do not establish the case for an overall beneficial or harmful effect resulting from a change in digoxin potency neither do they prove that changes in digoxin potency have no effect at all on mortality. It is possible that the increase in potency benefited some patients and harmed others and that the effects cancelled out. Several other countries introduced new standards for digoxin bioavailability at about the same time (1975). It would be useful to examine data from those countries for which monthly figures for cardiac deaths are available (as they are in England and Wales) and where the brands of digoxin which were widely available before the change were of low bioavailability. Data from any one country are likely to be affected by the extraneous factors already discussed while examination of those from several might uncover any small beneficial or harmful effect concealed in the present figures. Whatever the effect, it is unlikely to be large. A randomised controlled trial of the effects of digoxin on survival, despite its superior ability to control for other factors, would need to be extremely large to detect an effect that is not shown by these data.

\section{References}

1 Wilkins MR, Kendall MJ, Wade OL. William Withering and digitalis, 1785 to 1985 . $\mathrm{Br}$ Med f 1985; 290: 7-14.

2 Storstein L. Clinical and circulatory aspects of digitalis in heart failure. Eur Heart $\mathcal{f}$ 1982; 3 (suppl D): 59-64.

3 Dobbs SM, Kenyon WI, Dobbs RJ. Maintenance digoxin after an episode of heart failure: placebocontrolled trial in outpatients. $\mathrm{Br} \operatorname{Med} \mathcal{F} 1977$; i: 749-52.

4 Johnston GD, McDevitt DG. Is maintenance digoxin necessary in patients with sinus rhythm? Lancet 1979; i: 567-70.

5 Tunstall-Pedoe HD. Digoxin prescribing in general practice 1967-77. Lancet 1978; ii: 931-3.

6 Office of Population Censuses and Surveys. Registrar General's Statistical Review of England and Wales. Part I, Medical (years 1968 to 1973); Mortality Statistics England and Wales Series DH1, (years 1974 to 1976). London: Her Majesty's Stationery Office.

7 Bissell AF. Cusum techniques for quality control. Applied Statistics 1969; 18: 1-30.

8 Speizer FE, Doll R, Heaf P. Observations on recent increase in mortality for asthma. $\mathrm{Br} \mathrm{Med} \mathcal{F} 1968$; i: 335-9.

9 Danon A, Horowitz J, Ben-Zvi Z, Kaplanski J, Glick S. An outbreak of digoxin intoxication. Clin Pharmacol Ther 1977; 21: 643-6. 